\title{
ReaR
}

\section{¿Caminamos hacia el manejo del videolaringoscopios como primera opción en una vía aérea difícil?}

Artículo original: Cook F, Lobo D, Martin M, Imbert N, Grati H, Daami N, et al. Prospective validation of a new airway management algorithm and predictive features of intubation difficulty. Br J Anaesth. 2019 Feb;122(2):245-254. (릴ㅇ)

Correa Barrera JJ, San Juan Álvarez M, Gómez del Pulgar Vázquez B, Gholamian Ovejero S.

Hospital Universitario Severo Ochoa, Leganés, Madrid

\section{Resumen}

Determinar los factores predictivos de una vía aérea difícil constituye un reto para el médico anestesiólogo. La mayoría de guías actuales, sitúan los videolaringoscopios como elementos de rescate de una vía aérea fallida, tras una laringoscopia tradicional óptima. Establecer un algoritmo que en base a unas características físicas, permita determinar qué pacientes se beneficiarán del uso del videolaringoscopio como primera opción, puede suponer una ventaja y una disminución en los problemas relacionados con la vía aérea. Por otra parte, establecer cuáles de estos factores predicen con más fuerza una dificultad con el videolaringoscopio, nos ayudará a realizar mejores planes de abordaje y una óptima toma de decisiones sobre una vía aérea difícil. Este algoritmo ha sido capaz de conseguir la intubación traqueal de todos los pacientes en los que se ha previsto una laringoscopia difícil.

\section{Introducción}

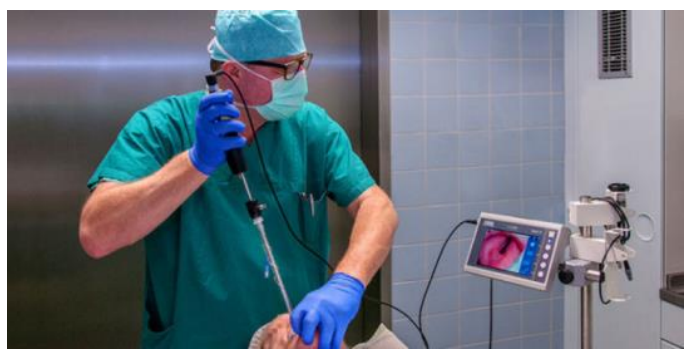

Determinar los factores predictivos de una vía aérea difícil constituye un reto para el médico anestesiólogo. La mayoría de guías actuales, sitúan los videolaringoscopios como elementos de rescate de una vía aérea fallida, tras una laringoscopia tradicional óptima. Establecer un algoritmo que en base a unas características físicas, permita determinar qué pacientes se beneficiarán del uso del videolaringoscopio como primera opción, puede suponer una ventaja y una disminución en los problemas relacionados con la vía aérea. Por otra parte, establecer cuáles de estos factores predicen con más fuerza una dificultad con el videolaringoscopio, nos ayudará a realizar mejores planes de abordaje y una óptima toma de decisiones sobre una vía aérea difícil. Este algoritmo ha sido capaz de conseguir la intubación traqueal de todos los pacientes en los que se ha previsto una laringoscopia difícil.

Entre los retos más importantes acerca del manejo de la vía aérea por parte del anestesiólogo en cirugías programadas se encuentra, sin duda, la de establecer un algoritmo que nos permita predecir de manera clara y sencilla a través de la evaluación de unos factores determinados, a los pacientes a los que se catalogarían como una vía aérea difícil. 
La mayor disponibilidad y extensión de los videolaringoscopios en la práctica clínica habitual se ha convertido en una realidad en la mayoría de centros, si bien a día de hoy estos dispositivos no se encuentran claramente encuadrados en los algoritmos de vía aérea publicados más habituales, por lo que la elaboración de esquemas de manejo propios de cada centro que nos ayuden a decidir el orden y el tipo de paciente que se beneficiará de su uso, puede constituir una mejora en la tasa de éxito del manejo de la vía aérea y en definitiva una disminución de los eventos adversos sobre la misma.

\section{Resumen del artículo}

El objetivo de este artículo es identificar los factores que predicen una dificultad de intubación orotraqueal por medio de la laringoscopia directa, estableciendo un algoritmo basado en dicha predicción que permite a los autores seleccionar los pacientes que se ven beneficiados del uso directo de un videolaringoscopios Airtraq $^{\mathrm{TM}}$, reduciéndose el daño secundario de intentos fallidos en la intubación tradicional.

A los siete factores usados habitualmente (apertura oral, Índice de Masa Corporal (IMC), grado de Mallampati, dificultad de intubación traqueal previa, perfil mandibular, distancia tiromentoniana y movilidad de la columna cervical), se les asigna una graduación entre 0 y 3 , siendo 0 la más baja y 3 la más alta. A todo paciente que obtenga la puntuación superior en un factor, o una puntuación $\geq 1$, en dos $o$ más factores, es asignado a la rama de uso directo de Airtraq ${ }^{\mathrm{TM}}$. El uso de un estilete y un fibroscopio se establece como "paso 2 y 3 " respectivamente en esta rama del siguiente algoritmo (Figura 1).

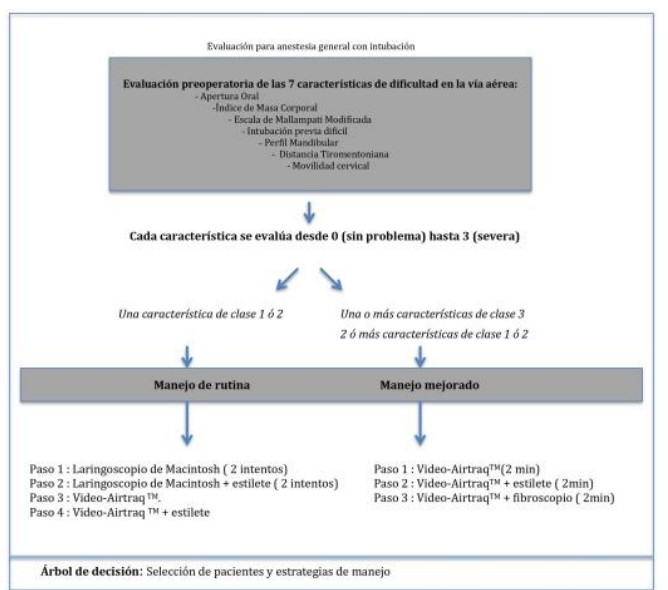

Figura 1. Árbol de decisión extraído/ traducido del artículo original.

Se realizó un estudio prospectivo, en el que la principal variable para ambas vías de manejo (Figura 1), fue el éxito en la intubación, el grado de intubación difícil (Pasos 1-4), y la incidencia de complicaciones del manejo de la vía aérea. Para el algoritmo mejorado además se consideró el tiempo de intubación traqueal y el porcentaje de apertura glótica. Las medidas de seguridad fueron la incidencia de desaturación arterial, el tiempo de intubación, y la presencia o ausencia de complicaciones graves.

Los resultados del modelo de predicción se calcularon utilizando la versión $R$ 3.2.4 R (R Foundation for Statistical Computing; Vienna, Austria) y los intervalos de confianza de incidencia de eventos adversos graves utilizando el método de Wilson.

Durante el periodo de estudio, se seleccionaron 16.695 pacientes, 15191 se asignaron manejo de rutina, donde se requirió el uso de estilete y Airtraq $^{\mathrm{TM}}$ en un 0,4 y $0,06 \%$ de los casos respectivamente. 1501 pacientes fueron asignados a la rama de manejo mejorado con uso directo de Airtraq ${ }^{\mathrm{TM}}$, todos ellos se intubaron con éxito. De estos, el $73 \%$ se intubó en menos de 30 segundos y solo un 4,5\% necesito de más de 4 minutos. Los pacientes que se intubaban en el paso $2 \mathrm{y}$ el paso 3 , 
dentro de la rama de manejo mejorado del algoritmo, que conllevan el uso de estilete y fibroscopio respectivamente, presentaban como factores predictivos más selectivos la restricción de apertura oral y disminución de la movilidad cervical.

El algoritmo mejorado permitió la intubación con éxito de todos los pacientes con laringoscopia difícil anticipada. La necesidad de combinar el uso de un estilete y un fibroscopio como el Airtraq ${ }^{\mathrm{TM}}$ se podía predecir con un alto grado de certeza.

Consideramos las $\begin{gathered}\text { siguientes } \\ \text { limitaciones } \\ \text { presentamos: }\end{gathered}$ del trabajo que

- La población del estudio es limitada: sólo se consideran cirugías programadas, quedando excluidas las urgencias, y no se incluyen pacientes obstétricos, pediátricos, pacientes con síntomas clínicos de reflujo, tumores de la vía aérea, ni traumatismos faciales y cervicales. A nuestro juicio estos subgrupos de pacientes pueden constituir un porcentaje importante de las vías aéreas difíciles previstas.

- La validez de los resultados del estudio únicamente es aplicable al videolaringoscopios Airtraq $^{\mathrm{TM}}$. Bajo nuestro punto de vista habría sido interesante añadir más ramas con otros dispositivos ópticos, lo cual haría más consistentes los resultados del estudio.

- El uso del algoritmo lleva asociado un aprendizaje tanto en simulación como en la práctica clínica habitual en el manejo del videolaringoscopios y el fibroscopio. Esta simulación puede no estar disponible en todos los centros.

- En el centro hospitalario donde se desarrolla el estudio, parte de las intubaciones tradicionales son llevadas a cabo por personal de enfermería debidamente entrenado. Esto puede añadir una dificultad para extrapolar ciertos resultados a nuestro país, donde dicha práctica no es habitual.

\section{Discusión}

El fallo en el manejo de la vía aérea se ha identificado como la primera causa de mortalidad y morbilidad relacionada con la anestesia. Al menos uno de cada tres accidentes relacionados con la anestesia se deben a dificultades en el control de la vía aérea ${ }^{(1)}$.

Sigue sin existir un consenso claro en la literatura sobre la definición de vía aérea difícil. La intubación difícil se define por la American Society of Anesthesiologists (ASA) como la situación en la que, con la cabeza en posición neutra y con manipulación laríngea externa, la introducción del tubo en la tráquea precisa más de diez minutos, requiere más de dos o tres intentos, o material accesorio al laringoscopio Macintosh ${ }^{(2)(3)}$. Una definición más cercana a la realidad clínica e independiente del tiempo es la situación en la que un anestesiólogo experimentado usando laringoscopia directa requiere más de 2 intentos con una misma pala, un cambio de pala o una ayuda para realizar la laringoscopia como una guía o un fiador ${ }^{(3)}$.

Actualmente no existe ningún test clínico ni radiológico que, aisladamente, sea capaz de predecir una intubación difícil. Existen multitud de test predictivos en forma de modelos multivariantes, entre ellos el de ElGanzuori y cols, Shiga y cols, Eberhart y cols, Wilson y cols, etc ${ }^{(4)}$. Cada uno de ellos evalúa una serie de factores lo que aporta a cada test predictivo distintas sensibilidades, especificidades $\mathrm{y}$ valores predictivos positivos.

Los videolaringoscopios son dispositivos ideados para mejorar la 
intubación traqueal. Constan de microcámaras y monitores portátiles de pantalla plana cuya intención es mejorar la visión y por tanto, el éxito de la laringoscopia directa. Existen metanálisis ${ }^{(5)}$ que comparan la laringoscopia directa con los videolaringoscopios, y que han reportado, para estos últimos, una mejor visión de la laringe, mayor tasa de éxitos en la intubación traqueal y mayor frecuencia de éxito con el primer intento, no describiéndose diferencias en el tiempo, dolor en la orofaringe, desviación de la columna cervical ni traumatismo sobre la vía aérea, labio, lengua y dientes.

Los videolaringoscopios ofrecen además un importante potencial para el manejo de la vía aérea en las áreas alejadas de quirófano, como la UCI o urgencias. Sin embargo, el gran número de dispositivos existente complica la realización de estudios que permitan llegar a un consenso sobre su incorporación en estos entornos remotos ${ }^{(6)}$.

El algoritmo de la ASA, incluye el uso de videolaringoscopios en el caso de ventilación con mascarilla facial adecuada e intubación fallida. No estableciendo un número máximo de intentos con estos dispositivos ${ }^{(2)}$. En este sentido, el algoritmo presentado introduce una mejora, ya que sitúa un escenario no recogido en la ASA, que no es otro que reconocer como primera posibilidad la intubación con videolaringoscopios de un paciente con determinados factores de dificultad, y dentro de esto poder incluso añadir más pasos con elementos como el estilete o el fibroscopio según cuales sean dichos predictores.

Aunque el uso de los videolaringoscopios ha sido mencionado en el algoritmo de manejo de la vía aérea difícil, con una evidencia tipo A por mejoría en la visualización laríngea, aún quedan controversias sobre el valor de este dispositivo en el manejo de la vía aérea difícil y la seguridad que representa para los pacientes, por lo que su significado sigue siendo un tema de investigación ${ }^{(7)}$.

La novedad introducida por este artículo, no radica en la selección de predictores para clasificar una vía aérea, sino en la creación de un algoritmo directo de decisión que incorpora en base a los mismos, los pacientes que se beneficiaran directamente de un videolaringoscopios como primer paso en la intubación, añadiendo además que predictores a su vez constituirán un fenotipo de dificultad incluso usando el dispositivo óptico ( En este algoritmo concretamente el videolaringoscopios Airtraq ${ }^{\mathrm{TM}}$ ).

En el estudio, se consideran directamente para intubación con fibrobroncoscopia aquellos pacientes que tienen una distancia interincisiva menor de $2 \mathrm{~cm}$ o aquellos en los que la limitación de la flexión cervical limita la introducción de un dispositivo rígido, sin embargo se reconsidera para la rama de intubación con Airtaq ${ }^{\mathrm{TM}}$ aquellos pacientes que no cumplían estas dos características, por lo tanto a priori, el algoritmo permite realizar con seguridad intubaciones complejas en paciente dormido $\mathrm{y}$ disminuye el número de intubaciones en paciente despierto con los beneficios que esto supone, tanto en recursos, como en tiempo así como en ahorrar estrés y ansiedad en el paciente.

Con este algoritmo se refleja de una manera sistematizada, lo que se viene realizando de manera extendida en la práctica clínica habitual, es decir usar planes de intubación que conllevan como primer paso un dispositivo óptico en pacientes que acumulan malos 
factores de predicción o que tienen historia previa de VAD.

Sin duda, elaborar y adaptar algoritmos como el que presenta el artículo a nuestros centros en función del videolaringoscopios del que se disponga, suponen una interesante herramienta para manejar, clasificar y en definitiva añadir seguridad a todos los pacientes que vayan a ser sometidos a una cirugía programada con anestesia general e intubación orotraqueal.

\section{Bibliografía}

(1) Frova G, Sorbello M. Algorithms for dificult airway management: a review. Minerva Anestesiol 2009;75(4):201-9. [PubMed]

${ }^{(2)}$ L. Apfelbaum, C.A. Hagberg, R.A. Caplan, C. D. Blitt, R.T. Connis, D.G. Nickinovitch, et al. Updated by the Committee on Standards and Practice Parameters; previous update was developed by the American Society of Anesthesiologists Task Force on Difficult Airway Management. Practice guidelines for management of the difficult airway: an updated report by the american society of anesthesiologists task force on management of the difficult airway. Anesthesiology. 2013 Feb;118(2):251-70. [PubMed]

(3) Practice guidelines for management of the difficult airway: an updated report by the American Society of Anesthesiologists task force on management of the difficult airwayAnesthesiology 2003, 98 (5): 1269-1277. [PubMed]
(4) Shiga T, Wajima Z, Inoue T, Sakamoto A. Predicting difficult intubation in apparently normal patients: a meta-analysis of bedside screening test performance. Anesthesiology 2005; 103(2): 429 -437. [PubMed]

(5) Jungbauer A, Schumann M, Brunkhorst V, Börgers A, Groeben H. Expected difficult tracheal intubation: A prospective comparison of direct laryngoscopy in 200 patients. $\mathrm{Br} \mathrm{J}$ Anaesth 2009; 102(4):546- 50. [PubMed]

${ }^{(6)}$ Neyrinck A. Management of the anticipated and unanticipated difficult airway in anesthesia outside the operating room. Curr Opin Anaesthesiol. 2013 Aug;26(4):481-8. [PubMed]

(7) J.B. Paolini, F. Donati, P. DroletReview article: Video-laryngoscopy: another tool for difficult intubation or a new paradigm in airway management?. Can J Anaesth. 2013 Feb;60(2):184-91. [PubMed]

Correspondencia al autor

Juan José Correa Barrera correabarrera83@gmail.com

Residente de $4^{\circ}$ año Anestesia y Reanimación. Hospital Universitario Severo Ochoa. Leganés, Madrid.

Mónica San Juan Álvarez sanjuanmo@gmail.com

FEA. Unidad de Anestesia y Reanimación Hospital Universitario Severo Ochoa. Leganés, Madrid.

Aceptado para el blog en enero de 2020 